\title{
Potential of the Surface Brightness Fluctuations method to measure distances to dwarf elliptical galaxies in nearby clusters
}

\author{
S. Mieske ${ }^{1,2}$, M. Hilker ${ }^{2}$, and L. Infante ${ }^{1}$ \\ 1 Departamento de Astronomía y Astrofísica, P. Universidad Católica, Casilla 104, Santiago 22, Chile \\ 2 Sternwarte der Universität Bonn, Auf dem Hügel 71, 53121 Bonn, Germany
}

Received 16 December 2002 / Accepted 23 January 2003

\begin{abstract}
The potential of the Surface Brightness Fluctuations (SBF) method to determine the membership of dwarf elliptical galaxies (dEs) in nearby galaxy clusters is investigated. Extensive simulations for SBF measurements on dEs in the $I$ band for various combinations of distance modulus, seeing and integration time are presented, based on average VLT FORS1 and FORS2 zero points. These show that for distances up to $20 \mathrm{Mpc}$ (Fornax or Virgo cluster distance), reliable membership determination of $\mathrm{dEs}$ can be obtained down to very faint magnitudes $-10>M_{V}>-12 \operatorname{mag}\left(\mu_{0}(V) \simeq 25 \mathrm{mag} \operatorname{arcsec}^{-2}\right)$ within integration times of the order of 1 hour and with good seeing. Comparing the limiting magnitudes of the method for the different simulated observing conditions we derive some simple rules to calculate integration time and seeing needed to reach a determined limiting magnitude at a given distance modulus for observing conditions different to the ones adopted in the simulations. Our simulations show a small offset of the order of 0.15 mag towards measuring too faint SBF. It is shown that this is due to loss of fluctuation signal when recovering pixel-to-pixel fluctuations from a seeing convolved image. To check whether our simulations represent well the behaviour of real data, SBF measurements for a real and simulated sample of bright Centaurus Cluster dEs are presented. They show that our simulations are in good agreement with the achievable $S / N$ of SBF measurements on real galaxies.
\end{abstract}

Key words. galaxies: clusters: general - galaxies: dwarf - galaxies: fundamental parameters - galaxies: luminosity function, mass fonction - galaxies: distances and redshift - techniques: photometric

\section{Introduction}

\subsection{The faint end of the galaxy luminosity function}

Dwarf elliptical galaxies (dEs) are the most numerous type of galaxies in the nearby universe, especially in clusters. This statement has been well established since the advent of CCD detectors and the building of telescopes with 4-8 $\mathrm{m}$ diameter that enabled observers to detect low surface brightness (LSB) objects substantially fainter than the night sky. With the improvement of observing facilities, the emphasis has over the last decade switched from detecting faint dwarf galaxies to quantifying well their properties and frequencies. Most of the times dEs are investigated in galaxy clusters, because for a cluster the distance and therefore the approximate angular size of candidate dwarf galaxies is known.

One of the most important statistical tools in investigating galaxy populations is the galaxy luminosity function $\Phi(M)$, describing the frequency of galaxies per magnitude interval. The knowledge of its logarithmic faint end slope $\alpha$ is very useful for testing models of galaxy formation. There are two fundamental

Send offprint requests to: $\mathrm{S}$. Mieske, e-mail: smieske@astro.puc.cl steps involved in determining the faint end of $\Phi(M)$ in galaxy clusters: first, finding the dwarf galaxy candidates; second, verifying that they are cluster members.

For the Local Group, $\Phi(M)$ has been determined down to $M_{V} \simeq-9$ mag (e.g. Mateo 1998; Pritchet \& van den Bergh 1999; van den Bergh 2000), suggesting $\alpha \simeq-1$.1. Local Group dwarf galaxies can nowadays readily be resolved into single stars with HST and/or active optics techniques. Thus, their distance can be determined; the second step in establishing $\Phi(M)$ is quite easy to perform. The first step, finding them, is more difficult due to their large angular extent and small contrast against the stars of the Milky Way. The latest discoveries of more and more faint dwarf spheroidals (e.g. Armandroff et al. 1999; Whiting et al. 1999) raise the question of how complete the Local Group sample is.

The opposite is true for nearby galaxy clusters. Here, finding candidate dwarf spheroidals is quite straightforward when performing deep enough photometry, but it is impossible to resolve them into single stars. For example, the brightest red giants of an early type galaxy at the Fornax cluster distance (19 Mpc, Ferrarese et al. 2000) have $V \simeq 29.4$ mag (Bellazzini et al. 2001). The confirmation of candidate dwarf spheroidals as cluster members must consequently be based on morphology 
and is therefore subject to possible confusion with background LSB galaxies. One depends on statistical subtraction of background number counts to estimate the faint end slope. Due to the generally low number counts, the Poisson error involved in this statistical subtraction constitutes a major source of uncertainty in determining $\alpha$, especially in magnitude-surface brightness bins where the contribution of background galaxies is of the order of, or higher than, that from cluster galaxies.

The majority of studies that have determined $\Phi(M)$ in nearby galaxy clusters (e.g. Sandage et al. 1985; Trentham et al. 2001, 2002a, 2002b and Hilker et al. 2003) suggest a logarithmic faint end slope of $-1.0>\alpha>-1.5$, being in substantial disagreement with CDM theory (Press \& Schechter 1974), which predicts $\alpha \simeq-2.0$ for the initial galaxy luminosity function (Kauffmann et al. 2000). Other authors, such as Phillips et al. (1998) for the Virgo cluster and Kambas et al. (2000) for the Fornax cluster, suggest a significantly steeper faint end slope of $\alpha \simeq-2$. This large discrepancy shows that much care must be taken when assigning cluster membership to galaxies for which no direct distance measurement is available. The Poisson statistics involved, especially when subtracting background number counts, can lead to different authors obtaining very different results for the same cluster.

Various methods for distance determination that can be applied to brighter galaxies outside the Local Group are not suited for the faintest dEs: standard candles such as SN Ia or Cepheids are very rare; radial velocity measurements, if possible, are very time consuming due to the large fields that have to be covered.

\subsection{Distances to galaxies with the SBF method}

An intriguing possibility to unambigously determine cluster membership of large samples of dEs in nearby clusters is deep wide field imaging and application of the Surface Brightness Fluctuations (SBF) method. The SBF-method was first described by Tonry \& Schneider (1988). SBF are caused by the fact that on a galaxy image the number of stars in each seeing disc is finite and therefore subject to statistical fluctuations. The amplitude of these fluctuations relative to the underlying mean surface brightness is inversely proportional to the number of stars. As the number of stars per unit angle increases quadratically with distance, the amplitude of the SBF is inversely proportional to distance and can therefore serve as a distance indicator.

As it is well known, the integrated light of an entire stellar population is dominated by the light emitted from the giants. In the case of an old population, only the red giants contribute. In the case of a younger population, there is also a contribution from the blue super giants. In the context of the SBF-method one can treat the whole stellar population as consisting only of stars having the mean luminosity weighted luminosity $\bar{L}=\frac{\int n(L) L \mathrm{~d} L}{\int n(L) \mathrm{d} L}$ of this population. The absolute surface brightness fluctuation magnitude $\bar{M}$ is then defined as $\bar{M}=-2.5 \times \log (\bar{L})+c$ with $c$ being the zero point of the photometric system. To obtain the distance modulus of a galaxy with the SBF-method, one directly measures the apparent SBF magnitude $\bar{m}$ and derives $\bar{M}$ from a distance-independent observable, usually a colour. Measuring the apparent magnitude and deriving its absolute value from a distance-independent observable is a procedure common to many distance determination methods, e.g. the P-L relation for Cepheids.

Tonry et al. (1997, 2000, 2001) have carried out an extensive survey to measure SBF for bright elliptical and spiral galaxies in 22 nearby galaxy groups and clusters within $40 \mathrm{Mpc}$. Using distances derived from cepheids or SN Ia in the respective galaxies, they obtain a relation between the absolute fluctuation magnitude $\bar{M}_{I}$ and the dereddened colour $(V-I)$, determined in the colour range $1.0<(V-I)<1.3$ :

$\bar{M}_{I}=-1.74+4.5 \times((V-I)-1.15)$ mag.

Up to now the SBF-method has only been applied to small samples of nearby dEs (e.g. Bothun 1991; Jerjen et al. 1998, 2000, 2001; Jerjen 2003).

\subsection{Aim of this paper}

In this paper, we focus on the potential of the SBF method to determine cluster membership of dEs in nearby clusters. We measure SBF of simulated sets of dEs at 3 different distance moduli between 7.6 and $48 \mathrm{Mpc}$ for various combinations of observing times and seeing. The paper is structured as follows: In Sect. 2, the impact of the age-metallicity degeneracy on SBF magnitudes is discussed. In Sect. 3, the technical details of simulating and measuring SBF are described and the properties of the different sets of simulated dEs are shown. In Sect. 4, the results of the SBF measurements for all simulated sets are presented and the limiting absolute magnitudes for cluster membership determination is discussed. A validity check of the simulations is presented by comparing real and simulated SBF data. We finish this paper with the conclusions in Sect. 5.

\section{Deriving $\bar{M}_{I}$ from $(V-I)$}

In the context of the SBF-method, the distance modulus of a galaxy is given by the difference between apparent and absolute fluctuation magnitude $\left(\bar{m}_{I}-\bar{M}_{I}\right)$. To estimate the reliability of the method, one must know both the accuracy in measuring $\bar{m}_{I}$ at the cluster distance and the uncertainty in deriving $\bar{M}_{I}$ for a $\mathrm{dE}$ with a given $(V-I)$.

In Fig. 1, theoretical values for $\bar{M}_{I}$ are plotted vs. $(V-I)$ for a set of old to intermediate age stellar populations with a wide range of metallicities, taken from Worthey ${ }^{1}$ (1994). Equation (1) is indicated as well. Note that Tonry et al. determined Eq. (1) only for $1.0<(V-I)<1.3$ as they used brighter and therefore redder galaxies. The theoretical and observed values for $\bar{M}_{I}$ agree quite well, although for $(V-I)>1.15$ the slope of Eq. (1) is slightly shallower than for the theoretical values.

Figure 1 shows that for $(V-I) \geq 1.0, \bar{M}_{I}$ depends mainly on $(V-I)$ and very little on the age-metallicity combination of the underlying stellar population. However, for $(V-I) \leq 1.0$

\footnotetext{
1 http://astro.wsu.edu/worthey/dial/dial_a_pad.html
} 
the spread in $\bar{M}_{I}$ at one given $(V-I)$, i.e. the effect of the agemetallicity degeneracy, rises significantly. At $(V-I) \simeq 0.85$ this leads to an uncertainty of up to 0.5 mag in relating $\bar{M}_{I}$ to $(V-I)$. Jerjen et al. $(1998,2000)$ have found an analogous spread of the order of 0.5 mag from $R$-band SBF measurements for blue dEs in the Sculptor and Centaurus A group. Additional colour information can help to reduce this uncertainty; e.g., at constant $(V-I)=0.9, \mathrm{~d} \bar{M}_{I} / \mathrm{d}(V-K) \simeq 10$ and $\mathrm{d} \bar{M}_{I} / \mathrm{d}(U-V) \simeq$ 5 (Worthey 1994). Precise photometry in $U, V$ and $I$ would therefore allow a precision of 0.1 to $0.2 \mathrm{mag}$ in deriving $\bar{M}_{I}$ from theoretical models.

While the age-metallicity degeneracy is an important source of uncertainty for distance measurements to blue field galaxies, it becomes useful as a relative age-metallicity indicator for blue cluster galaxies, as most of the time the cluster is separated from background/foreground galaxies by significantly more than $0.5 \mathrm{mag}$ in distance modulus. Spectroscopic surveys of the Fornax cluster (Drinkwater et al. 2000; Hilker et al. 1999) revealed a significant gap in radial velocity between Fornax members and background galaxies corresponding to $\simeq 3$ mag in $(m-M)$. Confusion of a blue and young background galaxy with a blue and old cluster galaxy is therefore very unlikely to happen.

Note that dIrr candidate members, to which the SBF-method is difficult to apply due to their irregular shape, can be distinguished morphologically in a straightforward manner from blue young background galaxies like anemic spirals. For clusters with a significant fraction of dIrrs, this morphological cluster membership assignment can complement the assignment based on SBF-distances for the smoothly shaped $\mathrm{dE}$ candidates and allow derivation of $\Phi(M)$ for the entire dwarf galaxy population.

\section{Simulating and measuring Surface Brightness Fluctuations in dEs}

We have simulated sets of dEs in the $I$-band with 3 distance moduli 29.4, 31.4 and $33.4 \mathrm{mag}$, corresponding to 7.6, 19 and $48 \mathrm{Mpc}$. This range was chosen to include distances to the more nearby groups like Leo I $(10 \mathrm{Mpc})$ as well as to the more distant clusters like Centaurus and Hydra (33 to 33.5 mag). Note that $31.4 \mathrm{mag}$ is the approximate distance modulus to Fornax and Virgo. The integration time was $3600 \mathrm{~s}$, the gain was 1 and the zero point $27.0 \mathrm{mag}$. The latter value is a mean of the VLT FORS1 and FORS2 zeropoint for imaging in the $I$-band when including an averaged colour term and extinction coefficient. The pixel scale was $0.2^{\prime \prime} /$ pixel, the image size $2048 \times 2048$ pixel. For each distance modulus, a set with $0.5^{\prime \prime}$ and $1.0^{\prime \prime}$ seeing was simulated. Additionally, for 31.4 mag distance modulus and $0.5^{\prime \prime}$ seeing, four different integration times were adopted, namely 900, 1800, 3600 and $7200 \mathrm{~s}$. Note that varying the integration time from $t_{1}$ to $t_{2}$ can also be considered as keeping the integration time fixed and adding $2.5 \log \frac{t_{2}}{t_{1}}$ to the zero point.

The photometric properties of our simulated dEs are derived from Hilker et al.'s (2003) values found for Fornax cluster dEs. For a given absolute magnitude $M_{V}$, the colourmagnitude- and surface brightness-magnitude relation from

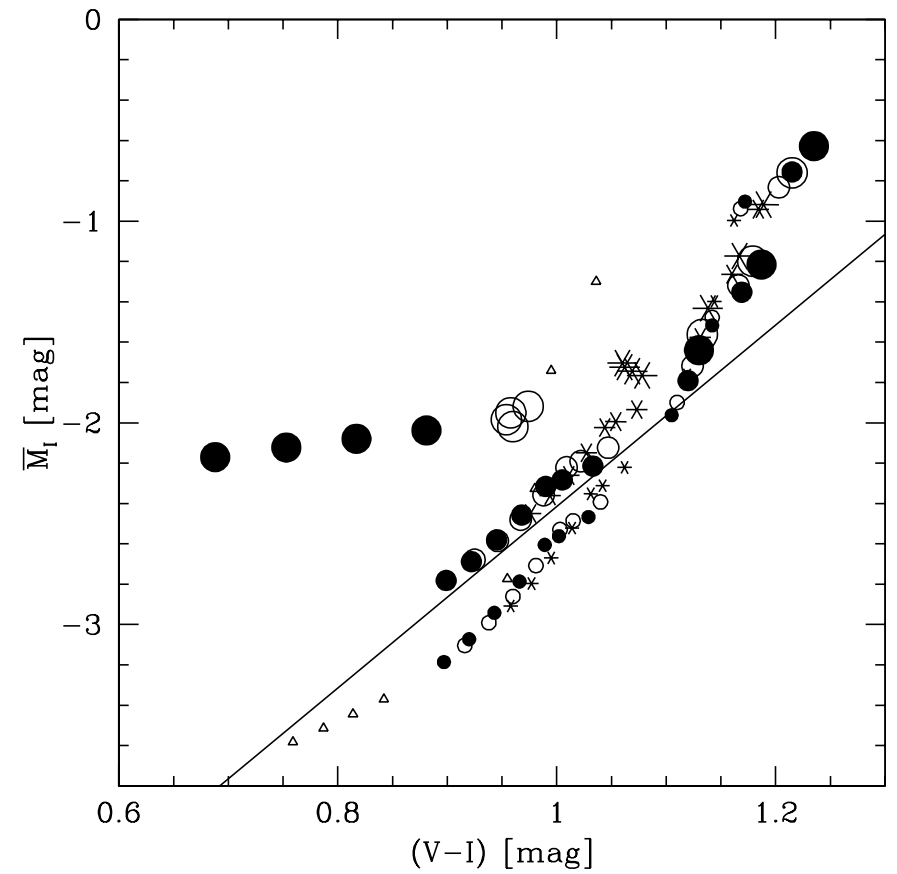

Fig. 1. Theoretical absolute fluctuation magnitude $\bar{M}_{I}$ plotted vs. the colour $(V-I)$ for a set of different stellar populations, as taken from Worthey (1994). The larger the symbols, the older the stellar population. Largest symbols: $17 \mathrm{Gyr}$; intermediate size symbols: $12 \mathrm{Gyr}$; small symbols: 8 Gyr (except for the open triangles, see below). Solid circles: Single burst populations with $\mathrm{Fe} / \mathrm{H}$ between $-1.7(-1.3$ for $17 \mathrm{Gyr}$ ) and 0 dex (left to right). Open circles and asterisks: $10 \%$ and $30 \%$ mixing of the single burst populations from above with a $5 \mathrm{Gyr}$ population of $\mathrm{Fe} / \mathrm{H}=0$. Open triangles: Single burst population of $1.5 \mathrm{Gyr}$ and $\mathrm{Fe} / \mathrm{H} \geq-1.3$. The solid line is defined by relation (1).

Hilker et al. (2003) is used to obtain $(V-I)$ and $\mu_{0}(V)$. For example, a dE with $M_{V}=-10$ mag will have $(V-I)=0.86 \mathrm{mag}$ and $\mu_{0}(V)=25.9 \mathrm{mag} \operatorname{arcsec}^{-2}$ while a dE with $M_{V}=-15 \mathrm{mag}$ will have $(V-I)=1.04 \mathrm{mag}$ and $\mu_{0}(V)=22.7 \mathrm{mag} \operatorname{arcsec}^{-2}$. An exponential intensity profile of the form $I(r)=I_{0} \times$ $\exp \left(-r / r_{0}\right)$ was adopted, with $r_{0}$ calculated from $M_{V}, \mu_{0}(V)$ and the adopted distance modulus. The ellipticity was chosen as zero. Globular Cluster (GC) systems are included, with a specific frequency of $S_{N}=5$ (Miller et al. 1998) for all galaxies, an absolute turnover magnitude $M_{I}=-8.5 \mathrm{mag}$ (Kundu \& Whitmore 2001) and the projected spatial GC density following the galaxy light distribution.

The final output of a SBF measurement procedure is the apparent surface brightness fluctuation magnitude $\bar{m}$ (in our case $\left.\bar{m}_{I}\right)$. This is equivalent to the luminosity weighted average apparent luminosity of the observed stellar population. As $\bar{m}_{I}=\bar{M}_{I}+(m-M)$, with $(m-M)$ being the adopted distance modulus of the simulated $\mathrm{dE}, \bar{M}_{I}$ determines the SBF amplitude at a given distance modulus. $\bar{M}_{I}$ was adopted as a function of $(V-I)$ according to Tonry's Eq. (1) for $(V-I)>1.0$. For $(V-I)<1.0$, it was decided to split the sample into two halves, since it is not known whether Tonry's Eq. (1) also holds for galaxies bluewards of this limit. For $50 \%$ of our galaxies, $\bar{M}_{I}$ was calculated according to Eq. (1). For the other $50 \%$ of 
our galaxies, $\bar{M}_{I}$ was kept constant at $\left.\bar{M}_{I}((V-I)=1.0)\right)=$ $-2.4 \mathrm{mag}$. Doing so we acknowledge that there is a significant age-metallicity degeneracy for $(V-I)<1.0$ (see Worthey's models). For the bluest galaxies simulated, with $(V-I) \simeq 0.8$ $\left(M_{V} \simeq-8.5 \mathrm{mag}\right)$, this implies a range in $\bar{M}_{I}$ of about $0.9 \mathrm{mag}$ between the two simulated samples. The effect for the simulations is that, on average, the SBF signal is weaker than if all dEs with $(V-I)<1.0$ were simulated according to Eq. (1).

To allow for varying seeing and integration times, the background field had to be created artificially. In a real background field obtained with VLT FORS 1 in the $I$-band at $3000 \mathrm{~s}$ integration, we fitted a power law distribution of the form $n(m)=A \times 10^{\gamma\left(m_{l}-m_{0}\right)}$ to the magnitude distribution of the objects detected by SExtractor down to the completeness limit of $I \simeq 25 \mathrm{mag}$. The fitted values were $A=14900 /$ degree $^{2}$, $\gamma=0.305, m_{0}=22$. According to this distribution, an artificial object field was created with the IRAF task mkobjects in the ARTDATA package, with $I=27$ mag as the faint limiting magnitude. Seeing and integration time were chosen as needed for the simulations. The sky brightness was adopted as $19.9 \mathrm{mag} \mathrm{arcsec}^{-2}$ in $I$, which holds within 3 days before and after new moon. To simulate large-scale flat-field effects, the background fields were multiplied by the normalized sky-map obtained from applying SExtractor to a VLT FORS1 flat field image.

Into each $2048 \times 2048$ pixel field, $16 \mathrm{dEs}$ were implemented. For each set with constant seeing, distance modulus and integration time, six fields were created, each with a different (random) spatial distribution of the background objects. This means that $96 \mathrm{dEs}$ were simulated for each set.

\subsection{Simulation of surface brightness fluctuations}

For each modelled pixel with a given distance $r$ to the galaxy center, first the number of stars corresponding to the surface brightness $\mu(r)$ of the exponential profile was calculated:

$N_{\text {stars } / \text { pixel }}(r)=10^{0.4 \times\left(\bar{M}_{I}+(m-M)-\mu(r)\right)} \times p^{2}$

with $p$ being the pixel scale. Then, a random number $N^{*}$ was chosen within a Poisson distribution centered on $N_{\text {stars/pixel }}(r)$. The intensity adopted at that pixel was then defined as

$I($ pixel,$r)=10^{-0.4 \times(\mu(r)-Z P)} \times p^{2} \times \frac{N^{*}}{N_{\text {stars } / \text { pixel }}(r)}$

with $Z P$ being the zero point, $27 \mathrm{mag}$ in our case. The implementation of the SBF is apparently achieved by multiplying by $\frac{N^{*}}{N_{\text {stars } / \text { pixel }}(r)}$. This means that along an isophote with radius $r$, the intensity $I(r)$ has a pixel-to-pixel rms of $\frac{I(r)}{\sqrt{N_{\text {stars } / \text { pixel }}(r)}}$. The image with the implemented SBF was then convolved with a Moffat seeing profile, which was modelled out to 7 times the $F W H M$. Finally, Poisson noise with rms $=\sqrt{I}$ was implemented. Once modelled, the galaxies were added onto the artificial background fields.

\subsection{Measurement of SBF}

To measure the SBF of a simulated $\mathrm{dE}$, the following steps were undertaken:

1. create object map with SExtractor of the whole image (containing $16 \mathrm{dEs}$ );

2. mask the dEs on the object map, subtract this image from original image;

3. create and subtract SExtractor sky map;

4. determine and subtract local sky level by a curve of growth analysis with the IRAF-task ELLIPSE in the ISOPHOTE package;

5. model mean galaxy light with ELLIPSE using a sigma clipping algorithm to disregard contaminating sources hidden below the galaxy light, subtract the model;

6. divide resulting image by square root of the model, cut out portion where SBF are measured;

7. mask out contaminating sources like foreground stars, background galaxies and globular clusters;

8. calculate the power spectrum (PS) of the cleaned image;

9. obtain the azimuthal average of the PS;

10. fit function of the form

$P(k)=P S F(k) \times P_{0}+P_{1}$

to the azimuthally averaged PS.

$P S F(k)$ is the PS of the seeing profile, normalized to unity at $k=0 . P S F(k)$ is determined from a simulated star with no close neighbours by fitting a Moffat profile to its PS. $P_{1}$ is the white noise component, proportional to the ratio between sky and galaxy brightness in the range where SBF were measured. It is independent of seeing. $P_{0}$ is the amplitude of the pixel-topixel surface brightness fluctuations, being the zero wavenumber limit of the seeing convolved pixel-to-pixel star count fluctuations, and therefore seeing-independent, too.

It holds that

$\bar{m}_{I}=-2.5 * \log \left(P_{0} / t_{\text {exposure }}\right)+Z P$.

Values at small $k$ (long wavelength) are rejected for the fit, as they are often considerably influenced by large-scale residuals from imperfect galaxy subtraction and the finite width of the image portion used to measure SBF.

The (seeing independent) $S / N$ of the measurement was defined as $S / N=P_{0} / P_{1}$, following Tonry \& Schneider (1988). In the following it will be referred to as canonical $S / N$. Note however that the detectability of SBF decreases with increasing seeing: by convolving with the seeing the star count pixelto-pixel fluctuations are smoothed out. Additionally, the larger the seeing is, the fewer the independent data points per unit angle that are available. To take this dependence of the SBF detectability on the seeing into account, we defined a modified signal to noise $S / N^{*}$ in the following way:

$S / N^{*}=P_{0} / P_{1} \times \sqrt{N_{\text {sd }}} / s f$

with $N_{\text {sd }}$ being the number of seeing discs - i.e. independent data points - contained in the image portion where SBF are measured, and $s f$ being the smoothing factor by which the seeing convolution reduces the pixel-to-pixel fluctuations. $s f$ was 5 for $0.5^{\prime \prime}$ seeing and 10 for $1.0^{\prime \prime}$ seeing, as the effective area of one seeing disc was 25 and 100 pixels, respectively. 


\subsubsection{Examples of simulated dEs}

In Fig. 2, example images and power spectra of simulated dEs are shown. The innermost pixels which were neglected in the power spectrum fit are especially marked. Note the effect of the twice as large seeing of $1.0^{\prime \prime}$ in the third example: the width of the seeing power spectrum is about half that of the other three examples, where the seeing was $0.5^{\prime \prime}$.

Rejecting the innermost pixels is crucial to determine the correct SBF amplitude, as low wavenumbers are affected by imperfect galaxy subtraction and large scale sky gradients in the investigated image. The limiting wavenumber beyond which one has to reject pixels has to be determined individually for each galaxy, as image dimensions and loci and number of contaminating sources change. We have adopted the following criterion for deciding which pixels to reject or not: if the $\chi^{2}$ of the fit improves by more than a factor of 2 when rejecting the innermost pixel, it is rejected. Then, the same is tested for the second pixel, and so on until $\chi^{2}$ improves by less than a factor of 2. For the examples given in Fig. 2, this criterion works fine for the lower three power spectra.

Unfortunately, as illustrated in the upper example, things can be more complicated. If only rejecting the inner- and outermost data point, the obtained fit fits well to wavenumbers smaller than 6 , but underestimates the signal for wavenumbers between 6 and 10 and overestimates the white noise component $P_{0}$. When rejecting wavenumbers smaller than 6 , the outer part is fit much better. The difference in $P_{1}$ between the two possibilities is considerable, about $40 \%$. In cases like that, the uncertainty in which pixels to reject or not is the major source of error. As is noted in Fig. 2's caption, the difference $\delta \bar{m}_{I}$ between simulated and measured $\bar{m}_{I}$ obtained when rejecting the inner 5 pixels is much smaller than for only rejecting the innermost pixel. Therefore, whenever fits to the outer and inner part of the power spectrum differed considerably, more emphasis was put on fitting well the outer part.

However, further out in the FT profile the white noise component starts to dominate over the PSF FT. As our simulations were performed on artificial dwarf galaxies, the image portions chosen for the SBF measurements had relatively small dimensions of typically 30 to 60 pixel (6 to $12^{\prime \prime}$ ). Therefore the wavenumber range over which the amplitude of the PSF is determined is only of the order of 10 or fewer independent data points. This small number, together with the uncertainty in which wavenumbers to disregard or not, is the major source of uncertainty for the SBF measurements we performed. For the simulated galaxies at $1.0^{\prime \prime}$ seeing, the wavenumber range over which to perform the fit to the PS is only half of that for 0.5 " seeing, which means the uncertainty at $1.0^{\prime \prime}$ seeing is significantly higher than for $0.5^{\prime \prime}$ seeing.

\section{Results of the simulations}

In Figs. 3 to 6, the results of the simulations are shown. Figures 3 to 5 show the results for the three different distance moduli with $3600 \mathrm{~s}$ integration time: $S / N^{*}$ and $\delta \bar{m}_{I}$ are plotted vs. $M_{V}$ for the two different seeing values. In Fig. 6, the same observables are plotted for four different integration times at a fixed 31.4 mag distance modulus and $0.5^{\prime \prime}$ seeing.

For the reasons mentioned in Sect. 3.2 we preferred to plot the modified signal to noise $S / N^{*}$ vs. $M_{V}$ instead of the canonical $S / N$, as $S / N^{*}$ takes into account seeing effects and thus allows a comparison between measurements obtained with different seeing. A 2nd order polynomial fit to $S / N^{*}$ vs. $M_{V}$ is plotted over the data points. The corresponding fit for the canonical $S / N$ is given as well. It is interesting to note that for $0.5^{\prime \prime}$ seeing the modified $S / N^{*}$ is consistently higher than the canonical $S / N$, while for $1.0^{\prime \prime}$ it is the opposite. This shows that for our simulations, $0.75^{\prime \prime}$ is about the limiting seeing below/above which $S / N^{*}$ becomes higher/lower than the canonical $S / N$.

One can see that for the highest $S / N^{*}$ data, the relative accuracy of the SBF method is of the order of $0.1-0.2 \mathrm{mag}$, i.e. 5-10\% in distance. By glancing at the bottom panels of Figs. 3 to 6 , one immediately notices an average offset between simulated and measured SBF amplitude of the order of $0.15 \mathrm{mag}$ in the sense of measuring too faint SBF. The mean offset, when disregarding the two most extreme offsets, is 0.14 mag. This offset is dealt with in more detail in Sect. 4.2.

\subsection{Limiting absolute magnitudes}

For each of the 9 simulated sets of dEs a limiting absolute magnitude $M_{V \text {,lim }}$ was determined, below which the determination of cluster membership is not reliable anymore. These are the two conditions for reliable cluster membership determination we adopted:

First, the difference between offset corrected measured and simulated $\bar{m}_{I}$ must be smaller than 0.5 mag. The mean offset with regard to which the measurement difference is defined is denoted as $\overline{\delta \bar{m}_{I}}$ and indicated in Figs. 3 to 6. The value of 0.5 mag was chosen as it is about equal to the maximum uncertainty in deriving $\bar{M}_{I}$ from $(V-I)$, see Sect. 2 . To require a higher measurement accuracy than the intrinsic uncertainty of the method would be unnecessary.

Second, the modified $S / N^{*}$ of the measurement must be higher than 6 . This limit was adopted to avoid an SBF measurement of an object with $S / N^{*}<4$ mimicking a $S / N^{*}$ of the order of 5 or 6 because of a measured $\bar{m}_{I} 0.5$ mag brighter than simulated.

$M_{V, \text { lim }}$ is then defined as the absolute magnitude at which $50 \%$ of the measured galaxies fulfil the above criteria. It is indicated for each set in Figs. 3 to 6 . As $\overline{\delta \bar{m}_{I}}$ and $M_{V \text {,lim }}$ depend on each other, they were determined iteratively.

In Table $1, M_{V, \text { lim }}$ is tabulated. One can see that for the two smaller distance moduli 29.4 and 31.4, the SBF-Method can reach very faint magnitudes. For galaxies with $M_{V} \simeq$ -10 to -11 mag within a distance of about $20 \mathrm{Mpc}$, reliable SBF measurements with accuracies better than 0.5 mag can be obtained at about $0.5^{\prime \prime}$ seeing, the given zeropoint of $27 \mathrm{mag}$ and an integration time of $1 \mathrm{hr}$. The SBF-Method is therefore a very valuable tool for extragalactic distance measurements, even for the faintest galaxies. 

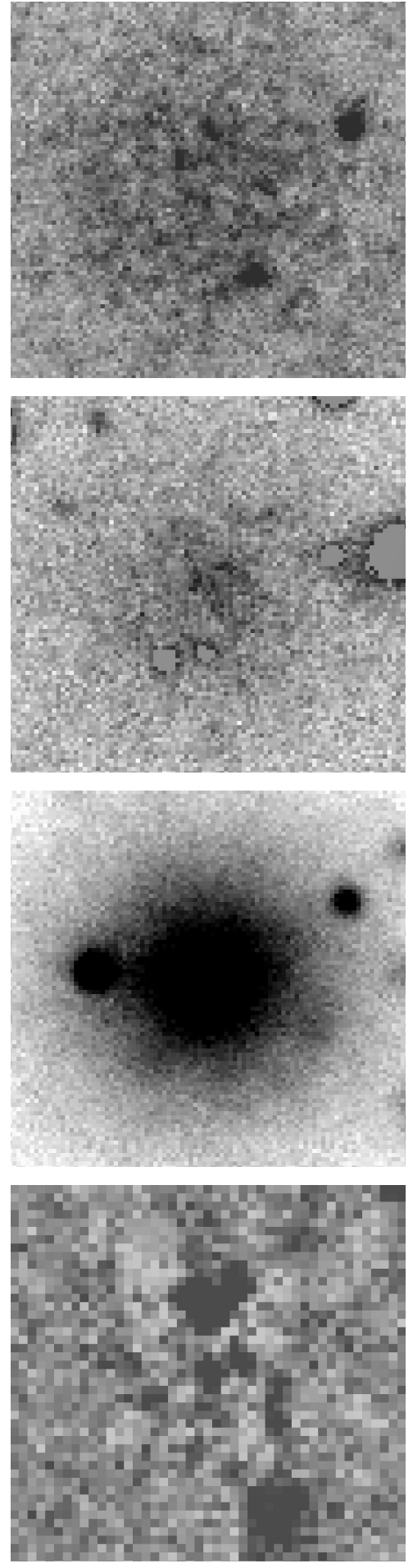
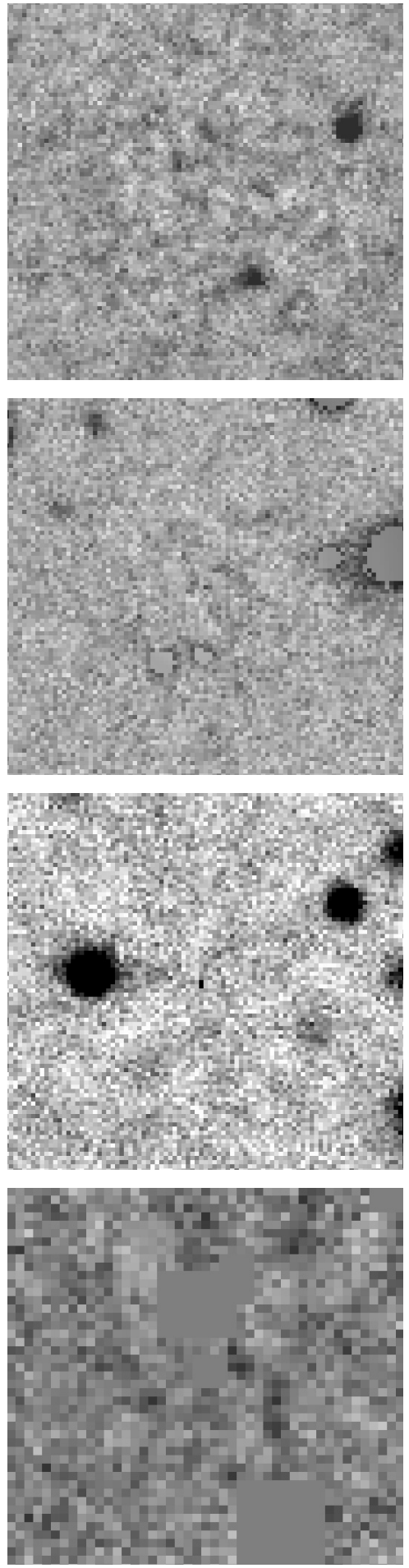
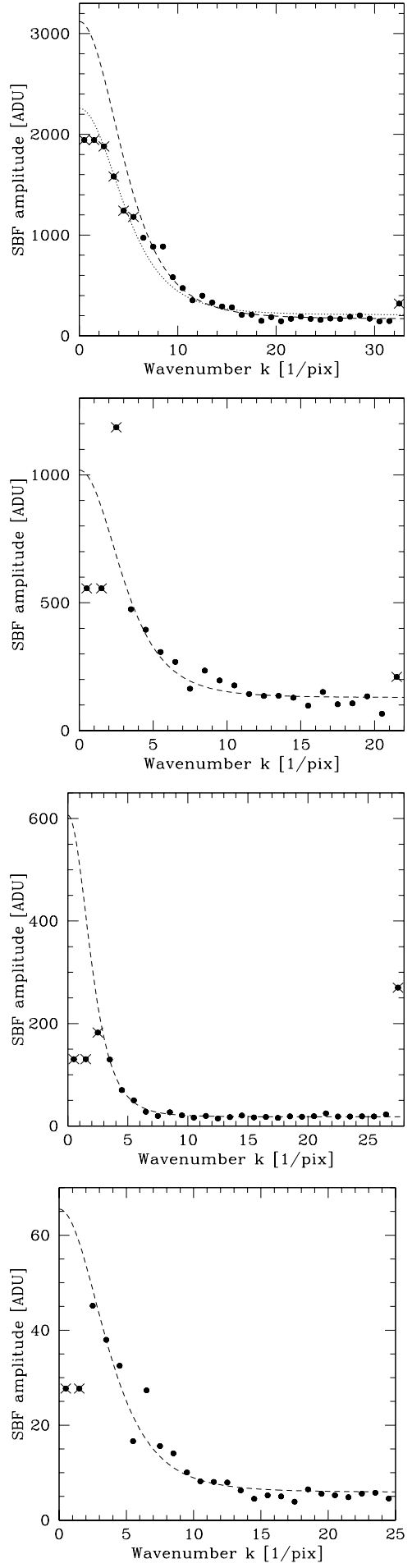

Fig. 2. Examples of simulated dEs in the $I$ band. For the three upper examples, the image sequence is: original image - model subtracted image - power spectrum of model subtracted, square root divided and cleaned image. For the lowest example, the sequence is: model subtracted image - cleaned model subtracted and square root divided image - power spectrum of the latter image. The different sequence for the lowest image was chosen to illustrate masking of contaminating point sources. For the power spectra, the dashed line is the best fit of $P(k)=P S F(k) \times P_{0}+P_{1}$, when rejecting the points marked with crosses. For technical reasons, the SBF amplitude at 0.5 wavenumber is equal to the amplitude at wavenumber 1.5. For the first example from the top, the dotted line represents a fit to all datapoints except the outermost and innermost one. The integration time was 1 hour for all examples. Parameters of the simulated dEs: 1 st panel from top: $M_{V}=-10.57 \mathrm{mag},(m-M)=29.4 \mathrm{mag}$, $\mu_{V}=25.5 \mathrm{mag} \operatorname{arcsec}^{-2},(V-I)=0.88 \mathrm{mag}, \bar{M}_{I}=-2.4 \mathrm{mag}, \delta \bar{m}_{I}=\bar{m}_{I \text {,simulated }}-\bar{m}_{I, \text { measured }}=-0.22 \mathrm{mag}$. Seeing $0.5^{\prime \prime}$. 2nd panel from top: $M_{V}=-11.13 \mathrm{mag},(m-M)=31.4 \mathrm{mag}, \mu_{V}=25.1 \mathrm{mag} \operatorname{arcsec}^{-2},(V-I)=0.90 \mathrm{mag}, \bar{M}_{I}=-2.86 \mathrm{mag}, \delta \bar{m}_{I}=0.06 \mathrm{mag}$. Seeing $0.5^{\prime \prime}$. 3rd panel from top: $M_{V}=-13.72 \mathrm{mag},(m-M)=31.4 \mathrm{mag}, \mu_{V}=23.42 \mathrm{mag} \operatorname{arcsec}^{-2},(V-I)=0.99 \mathrm{mag}, \bar{M}_{I}=-2.44 \mathrm{mag}, \delta \bar{m}_{I}=-0.02 \mathrm{mag}$. Seeing 1.0". 4th panel from top: $M_{V}=-16.74 \mathrm{mag},(m-M)=33.4 \mathrm{mag}, \mu_{V}=20.9 \mathrm{mag} \operatorname{arcsec}^{-2},(V-I)=1.10 \mathrm{mag}, \bar{M}_{I}=-1.98 \mathrm{mag}$, $\delta \bar{m}_{I}=-0.03$ mag. Seeing $0.5^{\prime \prime}$. 

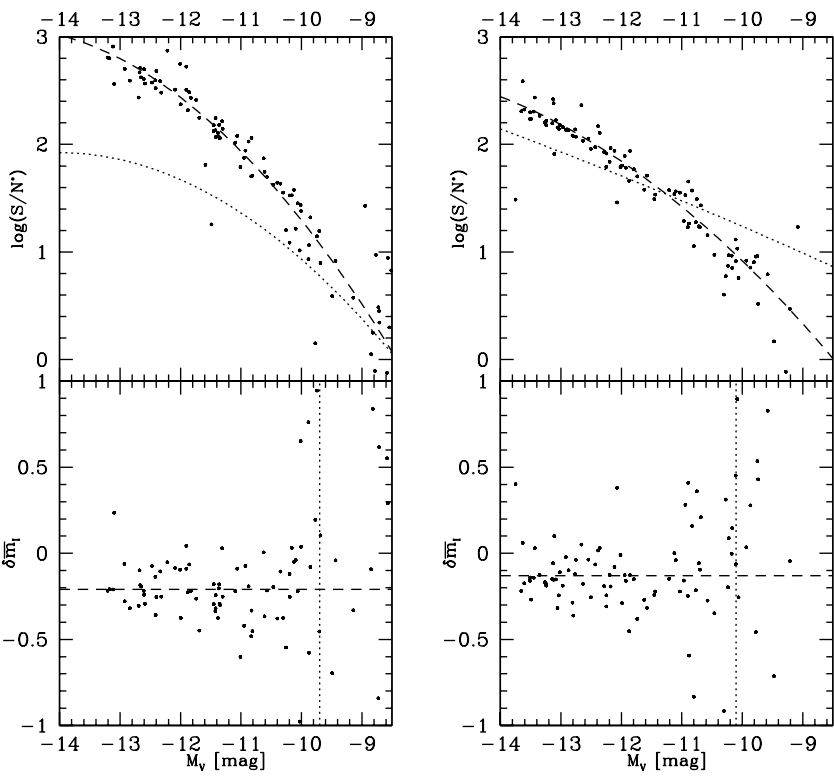

Fig. 3. Results of the simulations for $(m-M)=29.4 \mathrm{mag}$ and zero point $Z P=27.0+2.5 * \log (3600)$ mag. Left panel: seeing $=0.5^{\prime \prime}$. Right panel: seeing $=1.0^{\prime \prime}$. Top panel: Logarithm of $S / N^{*}$ (definition see text) plotted vs. $M_{V}$. The dashed line is a 2 nd order polynomial fit to the data points. The dotted line is the fit corresponding to the canonically defined $S / N$ (see text). Bottom panel: $\delta \bar{m}_{I}=$ $\bar{m}_{I, \text { simulated }}-\bar{m}_{I, \text { measured }}$ plotted vs. $M_{V}$. For magnitudes fainter than the limiting magnitude $M_{V}^{*}$ indicated by the dotted vertical line, more than $50 \%$ of the measured galaxies have $S / N^{*}<6$ or a deviation of more than $0.5 \mathrm{mag}$ from the horizontal dashed line. The latter one denotes the mean $\overline{\delta \bar{m}_{I}}$ for the measured galaxies brighter than $M_{V}^{*} \cdot \overline{\delta \bar{m}_{I}}$ and $M_{V}^{*}$ were determined iteratively.

Table 1. Limiting absolute magnitude $M_{V, \text { lim }}$ for the different combinations of distance modulus (denoted in the left column) and seeing (denoted in the top line). The integration time $t$ was $3600 \mathrm{~s}$ except for the four different values at $0.5^{\prime \prime}$ and 31.4 which correspond to four different integration times $t=900,1800,3600$ and $7200 \mathrm{~s}$ from left to right. The zero point including integration time $t$ is given by $27+2.5 * \log (t)$.

\begin{tabular}{lll}
\hline \hline & $0.5^{\prime \prime}$ & $1.0^{\prime \prime}$ \\
\hline 29.4 & -9.7 & -10.1 \\
31.4 & $-12.8 ;-11.7 ;-10.8 ;-10.8$ & -12.75 \\
33.4 & -14.9 & -16.75 \\
\hline
\end{tabular}

\subsubsection{Effects of varying seeing}

What are the effects of varying seeing, integration time and distance modulus?

Table 1 shows that for the distance moduli 31.4 and 33.4, increasing the seeing by a factor of 2 brightens $M_{V \text {,lim }}$ by about $2 \mathrm{mag}$. This corresponds to about $1.4 \mathrm{mag}$ in central surface brightness $\mu_{0}(V)$, or a factor of 3.6 in central intensity. This increase by almost a factor of 4 in central intensity is plausible: the pixel-to-pixel fluctuations are smoothed by a factor of 2 at $1.0^{\prime \prime}$ seeing compared to $0.5^{\prime \prime}$ while the SBF amplitude is
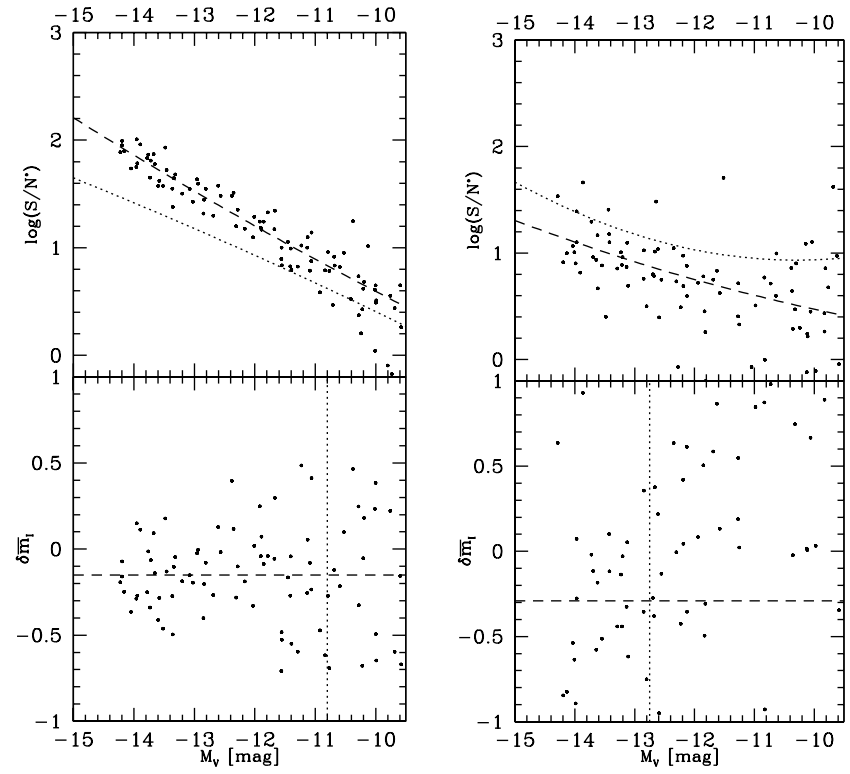

Fig. 4. Results of the simulations for $(m-M)=31.4 \mathrm{mag}$. Symbols/lines as in Fig. 3.
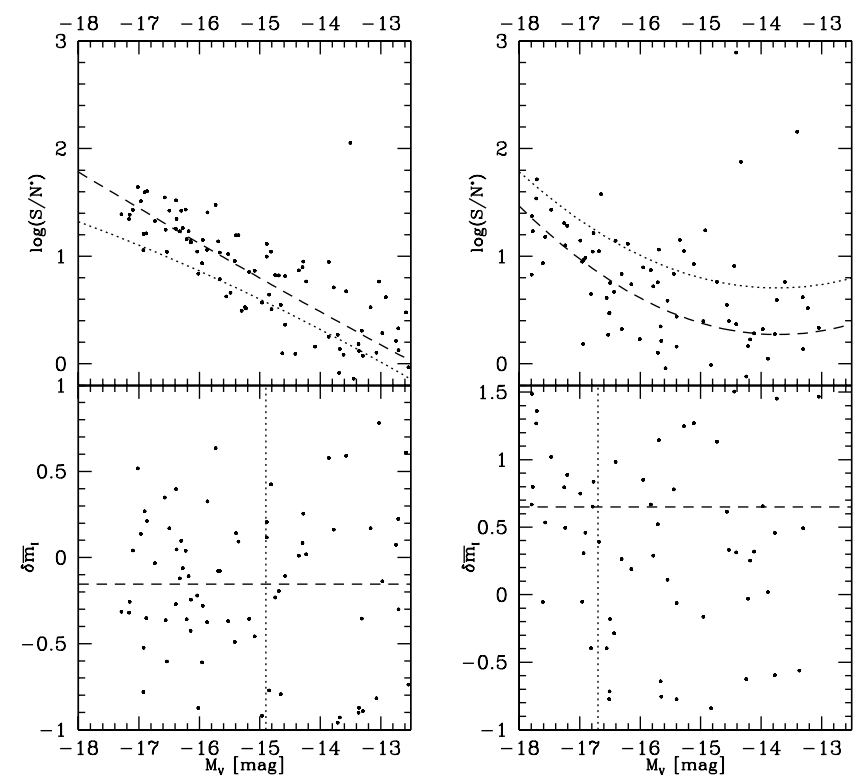

Fig. 5. Results of the simulations for $(m-M)=33.4$ mag. Symbols/lines as in Fig. 3.

proportional to the square root of the intensity; therefore the intensity must be increased by a factor of 4 to compensate for the smoothing caused by the twice as large seeing.

The "rule" extracted from that behaviour is: increasing seeing by a factor $x$ needs increase of intensity by a factor of $x^{2}$ to be compensated.

For the 29.4 mag distance modulus, there is no big difference between $0.5^{\prime \prime}$ and $1.0^{\prime \prime}$ seeing. This is because at the faint magnitudes around $-10 \mathrm{mag}$, the central surface brightness is about 25 mag $\operatorname{arcsec}^{-2}$ in $I$, which is only $2-3 \sigma$ above the sky standard deviation, i.e. the mean surface brightness is close to the detection limit, and measuring its fluctuations is 

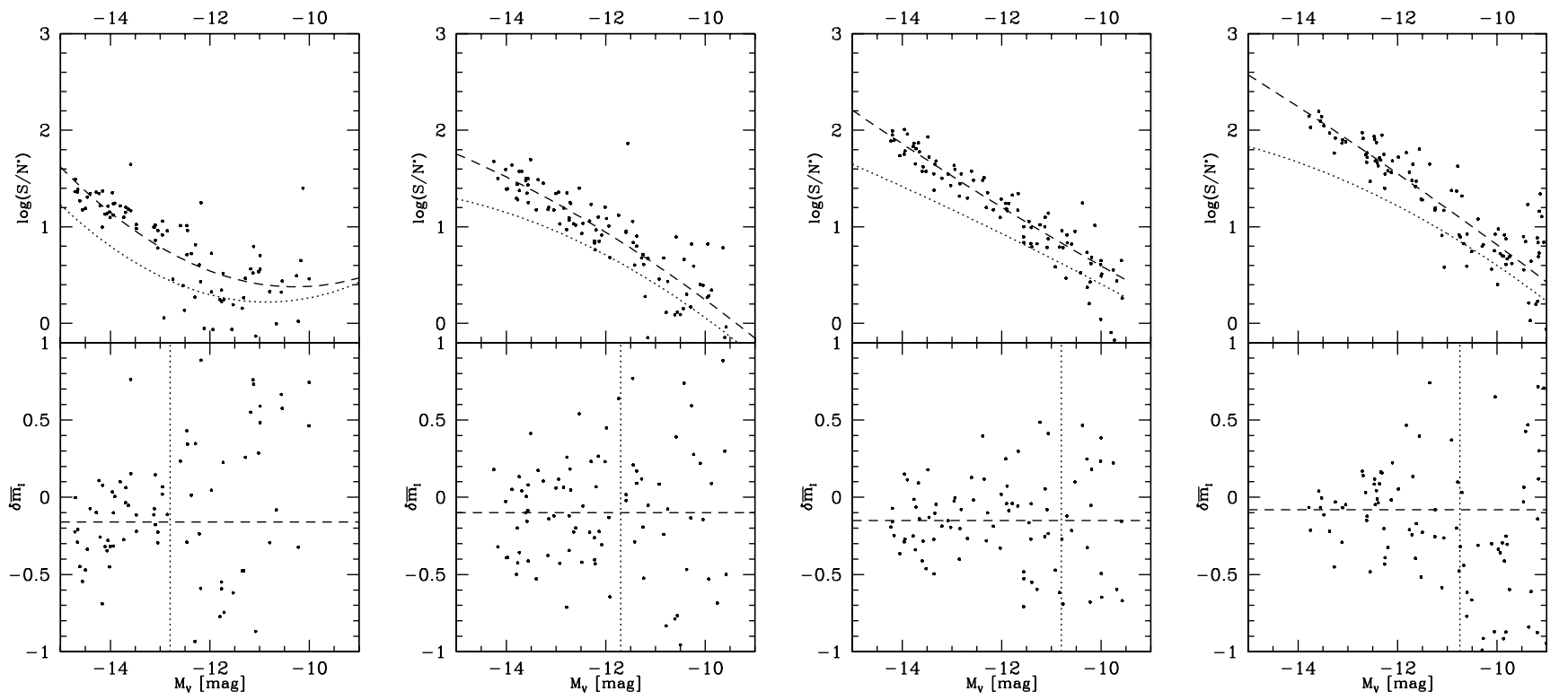

Fig. 6. Results of the simulations for $(m-M)=31.4$ and seeing $=0.5^{\prime \prime}$ at varying integration time / zero point $Z P=27.0+2.5 * \log (t)$. From left to right: $t=900 ; 1800 ; 3600 ; 7200 \mathrm{~s}$.

very difficult, even if the SBF amplitude is not much smaller than the mean surface brightness. That is why going from $1.0^{\prime \prime}$ to $0.5^{\prime \prime}$ seeing, no significant improvement of limiting absolute magnitude is reached for the 29.4 mag distance modulus.

\subsubsection{Effects of varying integration time / zero point}

What is the necessary scaling in integration time $t$ to account for varying seeing? At 31.4 mag distance modulus, only $t=$ $900 \mathrm{~s}$ are needed for $0.5^{\prime \prime}$ seeing to reach the same $M_{V \text {,lim }}$ as for $1.0^{\prime \prime}$ seeing and $t=3600 \mathrm{~s}$.

Thus, increasing seeing by a factor $x$ needs increase of integration time by a factor of $x^{2}$ to be compensated. This is equivalent to keeping integration time fixed and increasing the zero point by $2.5 * \log \left(x^{2}\right)$.

Table 1 shows that increasing the integration time $t$ by a factor of 2 results in a 1 magnitude fainter $M_{V, \text { lim }}$, or about a factor of 2 in central intensity. As $S / N$ and SBF amplitude are proportional to $\sqrt{t}$ and $\sqrt{I}$, respectively, this result should be expected.

Thus, increasing integration time $t$ by a factor $x$ allows SBF measurement for objects with central intensity fainter by the same factor $x$. This is equivalent to keeping integration time fixed and increasing the zero point by $2.5 * \log (x)$.

No notable change in limiting magnitude is seen when increasing $t$ from 3600 to $7200 \mathrm{~s}$. This might be partially due to statistical reasons, but the major reason is that the mean surface brightness is close to the detection limit (about 5 sigma above the sky noise) and the angular extent of the simulated dEs is only a few arcseconds. For galaxies with $M_{V} \simeq-11 \mathrm{mag}$, the additionally detected region when going from 3600 to $7200 \mathrm{~s}$ carries no measurable SBF signal anymore.

\subsubsection{Effects of varying distance modulus}

The strength of the SBF relative to the underlying mean surface brightness decreases linearly with distance. As the SBF are proportional to the square root of the intensity, the intensity must increase by a factor of $x^{2}$ when distance increases by a factor of $x$ to compensate for that. Table 1 shows that increasing distance modulus by 2 mag results in a 3-4 mag brighter limiting magnitude $M_{V, \mathrm{lim}}$. This corresponds to about a factor of 10 in central intensity, which is slightly more than the expected value of $2.5^{2}=6.25$. The reason for this is that the angular area over which the SBF signal is sampled is smaller at 2.5 higher distance for the same object.

\subsubsection{A simple rule}

Summarizing the scaling relations found in the last three subsections, we give the following rule to calculate the limiting magnitude $M_{V \text { new }}^{*}$ at new observing conditions different to the reference ones adopted in our simulations:

$M_{V \text { new }}^{*}=M_{V \text { ref }}^{*}-2.5 * 2.66 * \log \left(\frac{s_{\text {new }}}{s_{\text {ref }}}\right)-1.33 *\left(Z P_{\text {ref }}-Z P_{\text {new }}\right)(7)$

$M_{V \text { ref }}^{*}$ is the limiting magnitude calculated from our simulations at the given distance modulus. $s_{\text {ref }}$ is the seeing diameter in our simulations, $s_{\text {new }}$ the new seeing diameter. $Z P_{\text {ref }}$ is the total zero point in the simulations, i.e. $Z P_{\text {ref }}=27.0+2.5 * \log (t)$ with $t$ being the total integration time. $Z P_{\text {new }}$ is then the new total zero point. This all refers to a gain of 1, i.e. the zero point is expressend in terms of electrons and not ADU.

Note, however, that Eq. (7) is restricted to cases where the mean surface brightness of the galaxy is significantly higher than the sky noise. As when the surface brightness gets too close to the sky noise (less than about 5 sigma, see the former three subsections), changing integration time or seeing does not have strong effects on SBF detectability. 


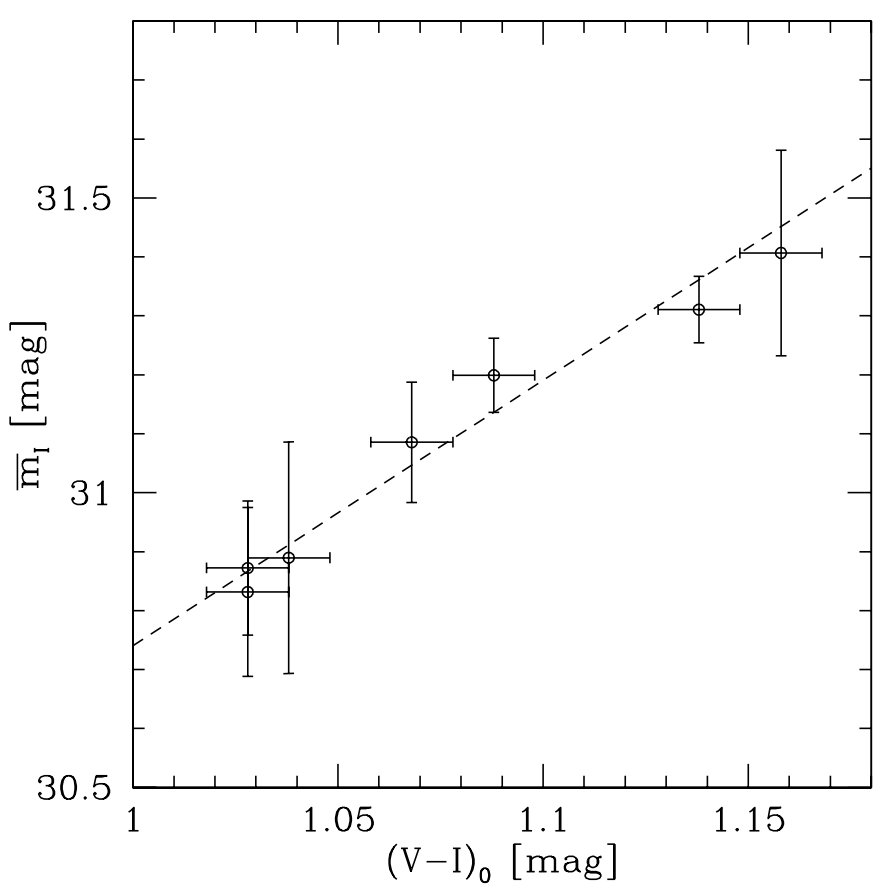

Fig. 7. Apparent SBF magnitude $\bar{m}_{I}$ plotted vs. $(V-I)_{0}$ for 6 Centaurus cluster dEs (from Mieske et al., in prep.). The bluest 2 data points correspond to the same galaxy imaged in 2 adjacent fields. The dashed line is a fit to the data when adopting a slope of 4.5, as in Tonry's Eq. (1). The corresponding distance modulus is $33.15 \pm 0.04$ mag. When fitting slope and zero point at the same time, the resulting slope differs by less than one $\sigma$ from 4.5 .

\section{2. $A$ bias in the simulations}

To find out the reasons for the 0.14 mag average offset between simulated and measured $\bar{m}_{I}$, a number of tests were performed:

First, an area of constant surface brightness and SBF amplitude was simulated, but not convolved with the seeing. After subtracting the mean brightness, dividing by its square root and calculating the power spectrum, the resulting image should have a mean equal to the SBF amplitude. This was the case to within $1 \%$, independent of surface brightness and SBF amplitude.

Second, an area of intensity zero was simulated with only one pixel given an intensity $I_{0}$ different from zero. This area was then convolved with the seeing profile. The mean of the resulting image was $0.99 \times I_{0}$. I.e. by restricting the seeing $\mathrm{psf}$ simulation to 7 times the $F W H M$, about $1 \%$ flux is lost. This is because we chose a realistic Moffat profile, which has stronger wings than the commonly used Gaussian.

The two above tests show that the algorithm used to implement and measure pixel-to-pixel SBF works correctly, and that convolution with the seeing profile to 7 times the FWHM imposes a negligibly small flux loss.

The final check performed was to simulate areas of several hundred pixel side length with constant surface brightness and SBF amplitude, which were then convolved with the seeing. After subtraction of the mean brightness, division by its square root, calculation of the power spectrum and azimuthally averaging, the SBF amplitude according to Eq. (4) - i.e. in the limit of $k=0$ - was too faint by 0.10 to $0.15 \mathrm{mag}$, independent of the

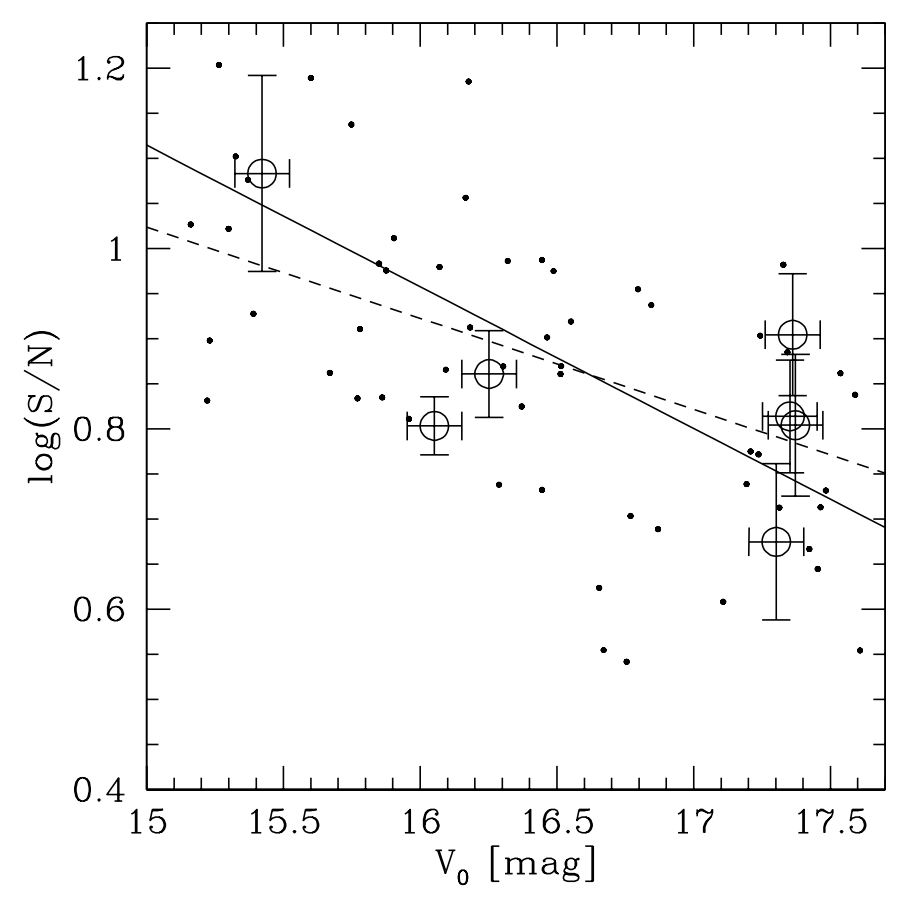

Fig. 8. $S / N$ of the SBF measurement vs. apparent $V_{0}$. Large circles are the real Centaurus data (from Mieske et al., in prep.). Dots represent galaxies simulated according to the Centaurus data's colour-SBF relation, colour-magnitude relation and surface brightness-magnitude relation. The dashed line represents a fit to the real data, the solid line is a fit to the simulated data. Both fits are consistent with each other within the error ranges.

strength of the simulated SBF, and for both seeing values 0.5 and $1.0^{\prime \prime}$. This offset corresponds to the one found in the main simulations. It shows that recovering the underlying pixel-topixel fluctuations from seeing convolved images is subject to small, but non-negligible loss of fluctuation signal, at least in our simulations.

Already Tonry \& Schneider (1988) noted a bias towards measuring too faint SBF of the order of $10 \%$ in distance or 0.2 mag in distance modulus, and attributed this to truncation of the seeing profile in their simulations. As mentioned above, in our case seeing truncation imposes a negligible flux loss.

Zero point problems of the order of $0.15 \mathrm{mag}$ are certainly a matter of concern when one aims at determining absolute values like $H_{0}$. However, our aim was to determine limiting magnitudes and surface brightnesses for cluster membership determination of dEs when using the SBF-Method. These measurements all rely on relative distances and are therefore independent of any zero point offset. The offset found in our simulations does not significantly change the statements made in the former sections.

We note that unresolved background galaxies generally increase the measured SBF signal. However, for our simulations this contribution is negligible. Using formula (13) of Jensen et al. (1998) for the $I$-band and inserting the values used for the magnitude distribution of background objects, we get for the relative contribution of background galaxies to the SBF signal at 33.4 distance modulus a value of the order of $2-4 \%$, 
depending on seeing and the galaxy's magnitude. For the distance moduli 29.4 and 31.4 , the contribution is below $1 \%$.

\subsection{Comparing real SBF data with simulations}

The $S / N$ achieved in SBF measurements for 6 bright Centaurus cluster dEs from VLT FORS1 images is compared with the $S / N$ obtained from simulations tuned to reproduce the measured values.

Figure 7 shows the colour-SBF relation found for the 6 galaxies (Mieske et al., in prep.). They cover a magnitude range of $15.4<V_{0}<17.4 \mathrm{mag}$. The colour-SBF relation from Fig. 7, a colour-magnitude relation and surface brightnessmagnitude relation were fit to the measured values of the Centaurus dEs, and 64 galaxies were simulated according to these relations. Their SBF amplitude was measured as described in Sect. 3. In Fig. 8, the $\log (S / N)$ values of the real measurements are plotted over the results for the simulations. A line is fit to both real and simulated data. One can see that the simulations do not overestimate the $S / N$ of the real data. Both fits are consistent with each other.

This consistency between real and simulated data confirms that the simulations presented in the previous sections are a good approximation of reality. The applied generalizations like zero ellipticity and purely exponential profile apparently do not introduce a notable bias towards too high or too low $S / N$.

\section{Summary and conclusions}

Extensive simulations of SBF measurements on dEs for three different distance moduli 29.4, 31.4 and 33.4 mag, two different seeings $0.5^{\prime \prime}$ and $1.0^{\prime \prime}$ and four different observing times 900 , 1800,3600 and $7200 \mathrm{~s}$ have been presented. For each of the simulated sets of dEs, the limiting magnitude $M_{V \text {,lim }}$ below which a distance measurement is not reliable anymore has been determined. It was shown that for distances $\leq 20 \mathrm{Mpc}$, the SBF method can yield reliable cluster membership of dEs down to very faint limiting magnitudes, e.g. $M_{V, \lim } \simeq-10$ mag for a distance of $7.5 \mathrm{Mpc}$, and $M_{V \text { lim }} \simeq-11 \mathrm{mag}$ for $19 \mathrm{Mpc}$ distance, at $1 \mathrm{hr}$ integration time, $0.5^{\prime \prime}$ seeing and a zero point of $27 \mathrm{mag}$ in the $I$-band. For the SBF measurements, a modified signal to noise $S / N^{*}$ has been defined that incorporates the seeing dependence of SBF detectability.

The effects of varying seeing, integration time and distance modulus on the limiting absolute magnitude are investigated. A number of simple rules, confirmed by theoretical considerations, are derived in order to calculate limiting magnitudes, needed integration times or seeing for observing conditions different to the ones adopted for our simulations.

It is pointed out that the total uncertainty in obtaining a distance modulus for a $\mathrm{dE}$ with SBF measurements is the quadratic sum of the measurement uncertainties for $\bar{m}_{I}$ and the uncertainties in deriving $\bar{M}_{I}$ from $(V-I)$. As both uncertainties are $0.5 \mathrm{mag}$ or smaller, the worst possible measurement accuracy is of the order of $0.65 \mathrm{mag}$ or $35 \%$ in distance. This would apply to very faint and blue dEs close to the limiting magnitude. For brighter and redder dEs, the total uncertainty decreases significantly, as deriving $\bar{M}_{I}$ from $(V-I)$ is less uncertain for red objects and the measurement accuracy improves to about $0.1 \mathrm{mag}$ for the brightest simulated dEs. While the uncertainty in deriving $\bar{M}_{I}$ from $(V-I)$ imposes a lower limit on the distance accuracy when observing field dEs, it allows rough age-metallicity estimates for blue cluster $\mathrm{dEs}$, as most of the times the cluster is separated from background/foreground galaxies by significantly more than $1 \mathrm{mag}$ in distance modulus.

We find that on average the measured SBF magnitude is 0.15 mag fainter than the simulated one. A number of tests show that this is due to loss of fluctuation signal when recovering pixel-to-pixel fluctuations from a seeing convolved image. This average offset shows that much care must be taken when deriving absolute values like $H_{0}$ with the SBF-Method. When aiming at relative distances like for cluster membership determination, a bias is of no concern.

By comparing real SBF data of Centaurus Cluster dEs with simulations tuned to reproduce the real data, we find that our simulations do not overestimate the achievable $S / N$ of the SBF method, but are consistent with real measurements. Therefore the statements about limiting magnitudes for the technique made in this paper are reasonable and we would not expect a very different behaviour in real observations.

An ideal application of the SBF technique would be a deep and wide field survey of several nearby clusters such as Fornax, Virgo or Doradus. With the arrival of wide field cameras on large telescopes (Suprime cam on the Subaru telescope or IMACS on Magellan), this is a very promising possibility to determine well the very faint end of the galaxy luminosity function in nearby clusters.

Acknowledgements. We thank the referee N. Trentham for his very helpful comments. SM was supported by DAAD PhD grant Kennziffer $\mathrm{D} / 01 / 35298$. LI acknowledges support by FONDAP Centro de Astrofísica No. 15010003. This work is partially based on observations obtained at the European Southern Observatory, Chile (Observing Programme 67.A-0358).

\section{References}

Armandroff, T. E., Jacoby, G. H., \& Davies, J. E. 1999, AJ, 118, 1220 Bellazzini, M., Ferraro, F. R., \& Pancino, E. 2001, ApJ, 556, 635

Bothun, G. D., Impey, C. D., \& Malin, D. F. 1991, ApJ, 376, 404

Drinkwater, M. J., Phillipps, S., Jones, J. B., et al. 2000, A\&A, 355, 900

Ferrarese, L., Ford, H. C., \& Huchra, J. 2000, ApJS, 128, 431

Hilker, M., Infante, L., Vieira G., Kissler-Patig, M., \& Richtler, T. 1999, A\&AS, 134, 75

Hilker, M., Mieske, S., \& Infante, L. 2003, A\&AL, 397L, 9

Jensen, J. B., Tonry, J. L., \& Luppino, G. A. 1998, ApJ, 505, 111

Jerjen, H., Freeman, K. C., \& Binggeli, B. 1998, AJ, 116, 2873

Jerjen, H., Freeman, K. C., \& Binggeli, B. 2000, AJ, 119, 166

Jerjen, H., Rekola, R., Takalo, L., Coleman, M., \& Valtonen, M. 2001, A\&A, 380, 90

Jerjen, H. 2003, A\&A, 398, 63

Kambas, A., Davies, J. I., Smith, R. M., Bianchi, S., \& Haynes, J. A. 2000, AJ, 120, 1316

Kauffmann, G., \& Haehnelt, M. 2000, MNRAS, 311, 576 
Kundu, A., \& Whitmore, B. C. 2001, AJ, 121, 2950

Mateo, M. L. 1998, ARA\&A, 36, 435

Mieske, S., \& Hilker, M., in preparation

Miller, B. W., Lotz, J. M., Ferguson, H. C., Stiavelli, M., \& Whitmore, B. C. 1998, ApJ, 508, L133

Phillipps, S., Parker, Q. A., Schwartzenberg, J. M., \& Jones, J. B. 1998, ApJ, 493, L59

Press, W. H., \& Schechter, P. 1974, ApJ, 187, 425

Pritchet, C. J., \& van den Bergh, S. 1999, AJ, 118, 883

Sandage, A., Binggeli, B., \& Tammann, G. A. 1985, AJ, 90, 1759

Tonry, J. L., \& Schneider, D. P. 1988, AJ, 96, 807

Tonry, J. L., Blakeslee, J. P., Ajhar, E. A., \& Dressler, A. 1997, ApJ, 475,399
Tonry, J. L., Blakeslee, J. P., Ajhar, E. A., \& Dressler, A. 2000, ApJ, 530,625

Tonry, J. L., Dressler, A., Blakeslee, et al. 2001, ApJ, 546, 681

Trentham, N., Tully, R. B., \& Verheijen, M. A. W. 2001, MNRAS, 325,385

Trentham, N., \& Hodgkin, S. 2002a, MNRAS, 333, 423

Trentham, N., \& Tully, R. B. 2002b, MNRAS, 335, 712

van den Bergh, S. 2000, PASP, 112, 529

Whiting, A. B., Hau, G. K. T., \& Irwin, M. J. 1999, AJ, 118, 2767

Worthey, G. 1994, ApJS, 95, 107 